\title{
Construction and Phenotypes of Double Sporulation Deficient Mutants in Streptomyces coelicolor A3(2)
}

\author{
By K. F. CHATER \\ Genetics Department, John Innes Institute, Colney Lane, Norwich $\mathrm{NR}_{4}{ }_{7} \mathrm{UH}$
}

(Received I8 September 1974; revised 25 November 1974)

SUMMARY

In whiA, B, G and $H$ mutants of Streptomyces coelicolor A3(2), aerial hyphae develop but sporulation septa are not formed. Septa are produced by whil mutants but are spaced abnormally far apart. Mutants in each locus have a distinctive aerial mycelium morphology, except for whiA and $B$ mutants which are closely similar. Seven strains were made with pairwise combinations of whi $A$ and $B$ mutations with whiG, $H$ and $I$ mutations and with each other. The genotypes of these strains were confirmed by suitable crosses and their aerial mycelium morphology examined. An indirect procedure was used to determine the aerial mycelium morphology of whiGH, GI and $H I$ double mutants. The double mutants always closely resembled one of the single mutant parent strains in morphology and a consistent scheme of epistasis was obtained - whiG being epistatic to whiH, $A, B$ and $I ;$ whiH to whiA, $B$ and $I$; and whiA or $B$ to whiI. These results point to the absence of any complex interactions between gene products, which might have been revealed by the occurrence of novel phenotypes in double mutants or by inconsistencies in the epistasis scheme.

\section{INTRODUCTION}

Previous papers have described the isolation, phenotypes and genetic mapping of white colony (whi) mutants of Streptomyces coelicolor A3(2) defective in the conversion of aerial hyphae into mature spore chains (Hopwood, Wildermuth \& Palmer, 1970; Chater, 1972; Chater \& Merrick, 1974; McVittie, 1974). Eight whi genes ( $A$ to $E, G$ to $I$ ) have been identified; the status of a ninth (whiF) is doubtful (Chater \& Hopwood, 1973; Chater \& Merrick, 1974). Of these, whi $A, B, G$ and $H$ mutants were apparently unable to form the special sporulation septa (Wildermuth \& Hopwood, 1970) required to subdivide the long aerial hyphal cells into spore-sized compartments; whil mutants produced aberrantly spaced sporulation septa (Hopwood et al. 1970); the single whiC mutant was oligosporogenous (Chater \& Merrick, I974); and whiF mutants were defective in the formation of mature spores from spore compartments (McVittie, I974). The frequency with which whi,$B$, $G, H$ and $I$ mutants occurred suggested that few if any other genes were specifically involved in the early stages of sporulation of aerial hyphae (Chater \& Hopwood, 1973).

Most of these mutants had aerial hyphal morphology resembling intermediate stages in spore formation in the wild type. The sequence in which these stages take place should be determinable by studies of the morphology of strains carrying two different whi mutations. In this paper I describe the construction and phenotypes of a set of double mutant strains involving the five genes whi $A, B, G, H$ and $I$. 


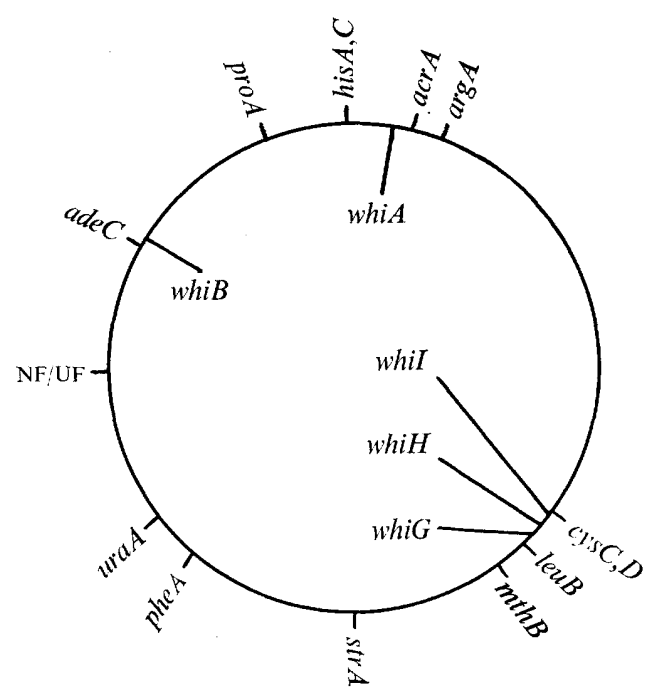

Fig. I. Linkage map of markers employed. The map and gene symbols are based on those of Hopwood et al. (1973). Morphological markers are given inside the circle.

\section{METHODS}

Strains. All strains (Table I) were originally derived from Streptomyces coelicolor A3(2) (Hopwood, 1959). The locations on the circular linkage map of all the markers employed are given in Fig. I.

Media, culture methods, mating techniques and light microscopy. These were all as described by Chater (1972), microscopy being done on impression preparations.

Fertility testing. Fertility tests to distinguish IF and NF types were as described by Vivian \& Hopwood (I970), with UF strains II90 or A200 as testers.

\section{RESULTS}

\section{Morphology of constructed double whi mutants containing whiA or whiB mutations}

The sections immediately following describe the synthesis of seven strains containing pairwise combinations of the two mutations whiAI70 and whiB2I 8 with whiG7I, whiH25O or whil8o, and with each other. The genetic confirmation of the presence of the pairs of whi mutations in each strain is also described. To facilitate understanding of some of the genetics, the phenotypes of the double whi mutants and the single mutant parent strains are described first.

The mapping and phenotypes of all five mutations were given by Chater (I972) and are summarized in Figs. I and 2 and in Table I. Each is typical of mutations of its locus; whiA and $B$ mutants usually have type II phenotype (non-fragmenting, often tightly coiled aerial hyphae); whiG mutants have type I phenotype (non-fragmenting aerial hyphae showing no coiling); whiH mutants have type III phenotype (non-fragmenting, loosely coiled hyphae); whil mutants have type IV phenotype (coiled, much-fragmented aerial hyphae).

Each double whi strain was found to resemble closely one of the single whi parent strains (Fig. 2, Table I). These data suggested that whiG and $H$ were epistatic to whi $A$ and $B$, and whi $A$ and $B$ to whil, while the whiAB double mutant was indistinguishable in phenotype from either single mutant parent strain. These conclusions were supported by data, given 

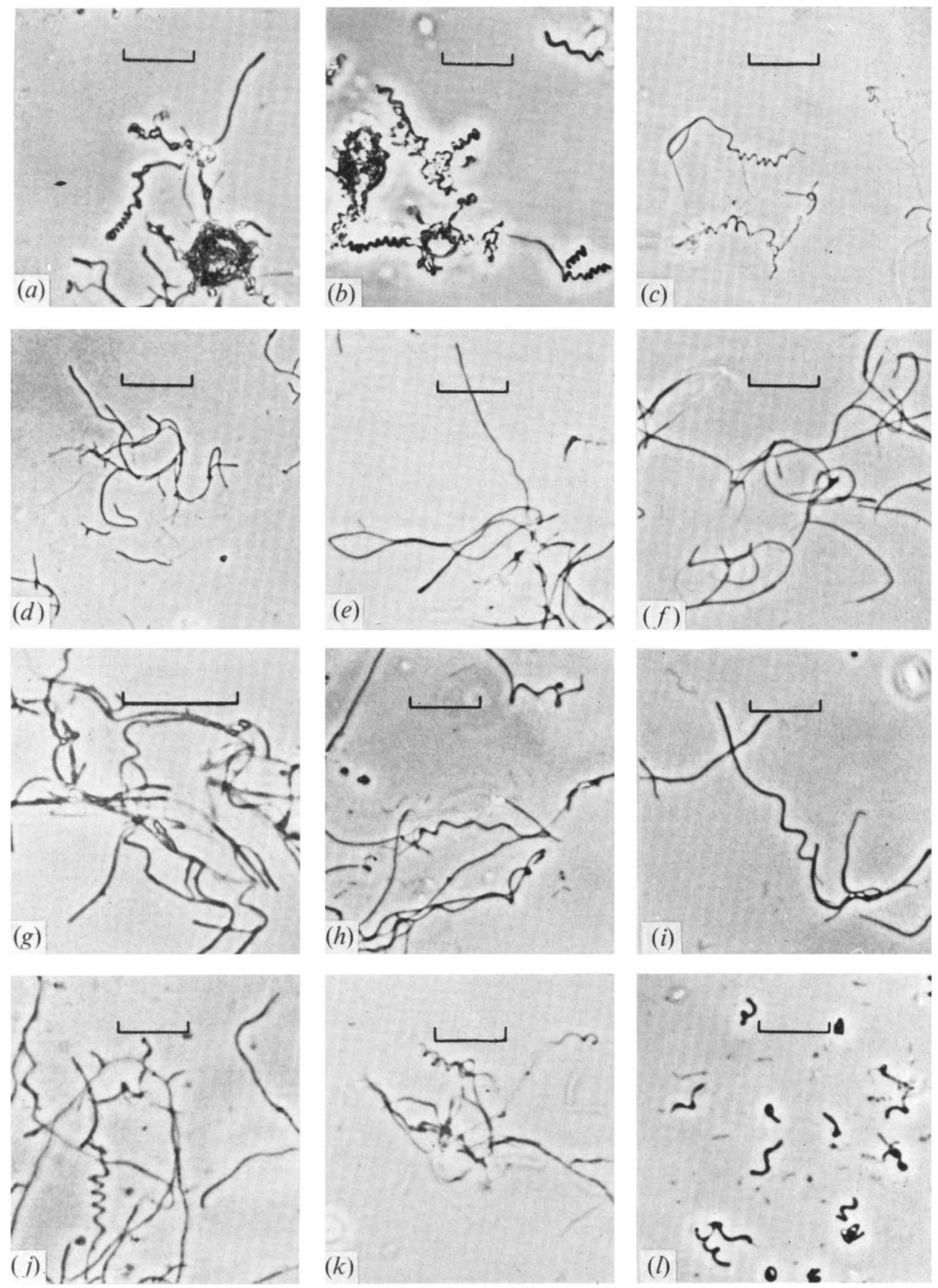

Fig. 2. Phase-contrast photomicrographs of impression preparations of the aerial hyphae of single and double whi mutants. All bar markers indicate $20 \mu \mathrm{m}$. (a) whiAr 70 (phenotype II); (b) whiB2I 8 (II); (c) whiAI7oB2I8 (II); (d) whiAI7OG II (I); (e) whiB2I8G7I (I); (f) whiG 7 I (I); (g) whiAI 7oH250 (III); (h) whiBzI8H250 (III); (i) whiH250 (III); (j) whiAI 7oI80 (II); (k) whiB2I8I80 (II); (l) whil8o (IV). Phenotypic designations (Roman numerals) are those of Chater (I972). 


\section{Table I. List of strains}

Explanations of symbols and fertility type designations are given in Hopwood, Chater, Dowding \& Vivian (1973). Morphological phenotypes are those given by Chater (1972) (see also Fig. 2).

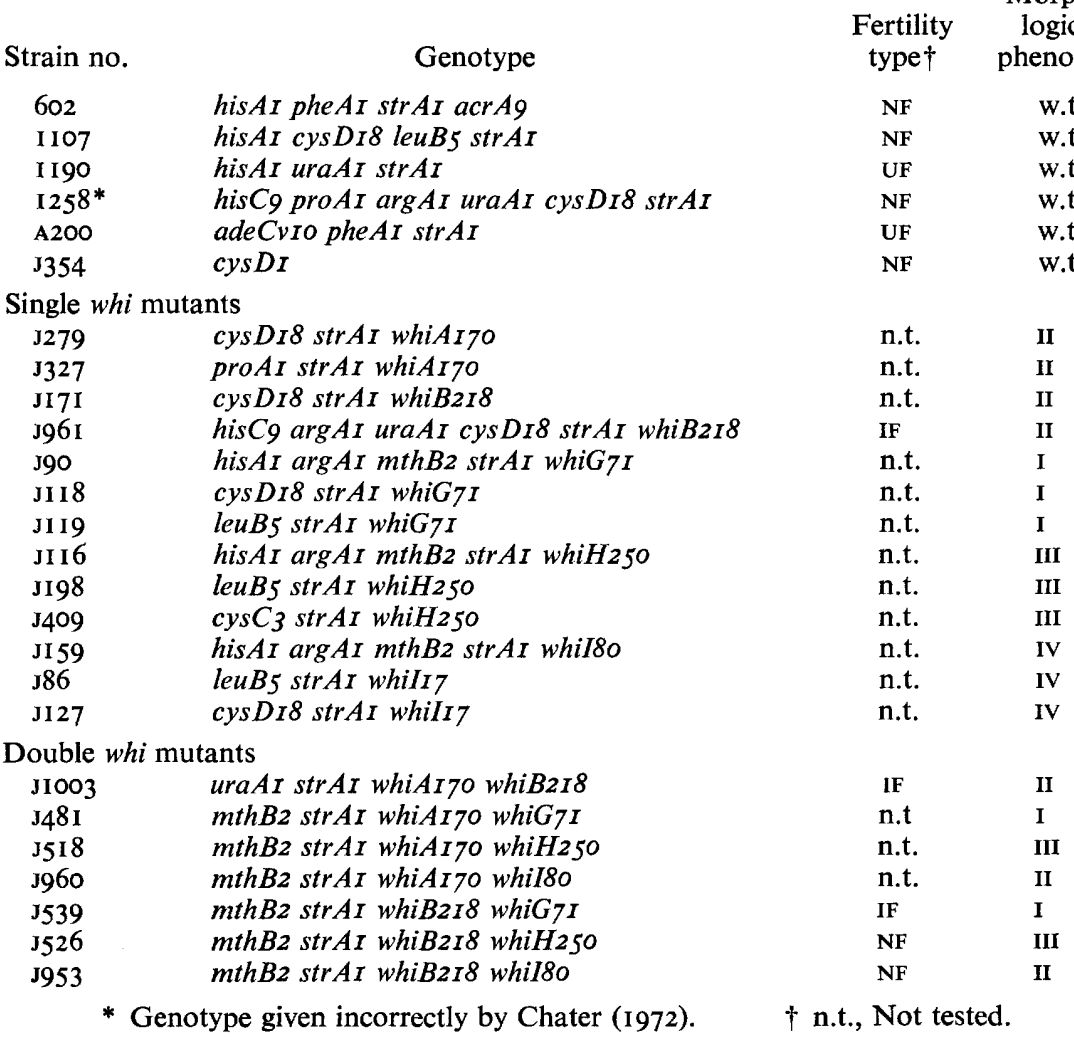

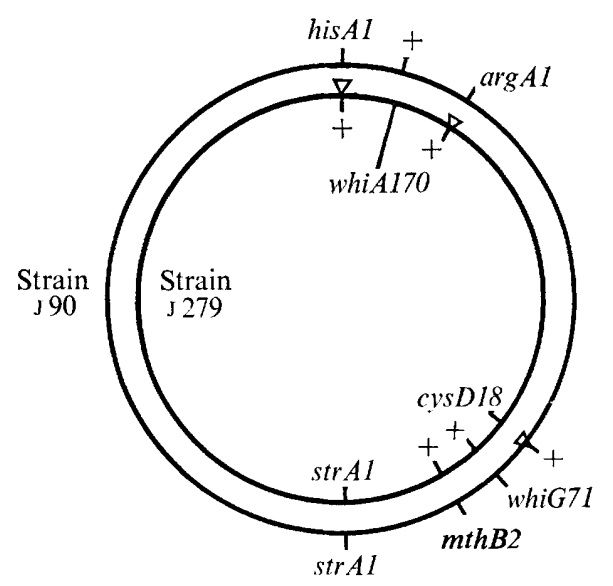

Fig. 3. Synthesis of a whiAG double mutant. Strains 190 and $\mathbf{3 2} 79$ were crossed and his $A^{+} \arg A^{+} \operatorname{cys} D^{+}$ recombinants selected (triangles). These were screened for homoserine-requirement (the $m t h B 2$ phenotype). A sample of $m t h B 2$ recombinants was then tested for inheritance of the two whi mutations as shown in Tables 2 and 3. Similar crosses, in which strain J90 was replaced by strains J I 6 or J159, were used to construct whi $A H$ and $A I$ double mutants. 


\section{Table 2. Analysis of a strain (J48r) carrying whiA and $G$ mutations}

Strain J48I was crossed with 1258 and selection (triangles) made for pro $A^{+} m t h B^{+}$recombinants. These were analysed for non-selective markers, and samples of whi recombinants re-streaked and examined microscopically. The phenotypic classification is that of Chater (1972) (see also Fig. 2). The frequency of each allele among the progeny is given in the diagram, the frequency of whi recombinants being determined by the inheritance of whiA (see text).

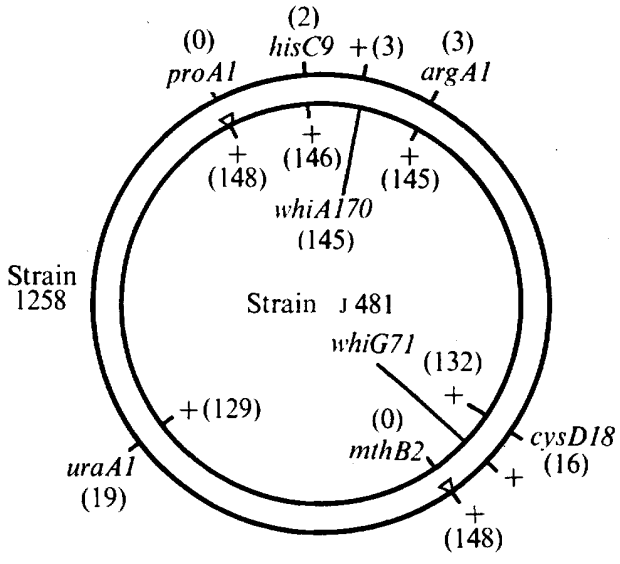

\begin{tabular}{|c|c|c|c|}
\hline \multirow[b]{2}{*}{ Genotype } & \multirow{2}{*}{$\begin{array}{l}\text { Frequency } \\
\text { of } \\
\text { recombinants }\end{array}$} & \multicolumn{2}{|c|}{$\begin{array}{l}\text { Phenotype of aerial mycelium } \\
\text { in a sample of recombinants }\end{array}$} \\
\hline & & Type I & Type II \\
\hline$u r a A$ whi & 12 & - & - \\
\hline$w h i$ & I 20 & 20 & 2 \\
\hline cys $D$ uraA whi & 4 & 0 & I \\
\hline cys $D$ whi & 8 & 0 & 4 \\
\hline $\arg A \operatorname{cys} D$ ura $A$ whi & I & o & I \\
\hline his $C \arg A$ cys $D$ uraA & I & - & - \\
\hline his $C \arg A$ cys $D$ & I & - & - \\
\hline hisC uraA cysD* & I & - & - \\
\hline & tal 148 & & \\
\hline
\end{tabular}

below, from crosses involving the double mutants, where the segregation of parental whi mutant phenotypes among whi recombinants was only explicable in terms of the epistatic relationships summarized above.

\section{Synthesis and testing of whiAG, AH and AI double mutants}

The diagrams and Tables relating to this section all describe in detail the situation for the whi $A G$ double mutant. To save space and preserve clarity, the methods and results for the whi $A H$ and $A I$ mutants will merely be summarized in the text.

Fig. 3 illustrates the type of cross used to synthesize these strains. The selection of strains requiring only homoserine from these crosses ensured that, unless multiple crossing-over had occurred, the recombinants would inherit both the whiA and the whiG, $H$ or $I$ mutation.

In the crosses in which the presence of both whi mutations in each double mutant was confirmed (Tables 2,3 ) the double whistrain was always counterselected at $m t h B 2$, the counterselection against the tester strains being such that the two whi mutations were not separated 
Table 3. Further analysis of a strain (J48I) carrying whiA and $G$ mutations

Strain $\mathrm{J} 48 \mathrm{I}$ was crossed with $\mathrm{I} 107$ and selection (triangles) made for $m t h B^{+} l e u B^{+}$recombinants.

For further explanation, see Table 2 and text.

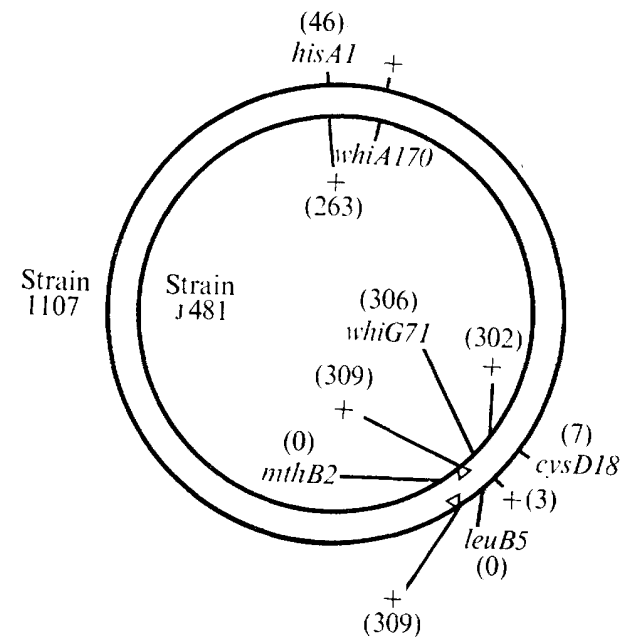

$\begin{aligned} & \text { Frequency } \\ & \text { of }\end{aligned}$
$\begin{aligned} & \text { Genotype } \\ & \text { recombinants }\end{aligned}$

by the selection points. As a result, when the segregation of white and grey colony colour was scored, a whi map location would be obtained corresponding to that of the whi mutation situated nearer to the counterselection point of the tester strain. In Table 2 this point was at proA, so that white colony colour segregated as if determined by whiA, while in Table 3 it was at $l e u B$, so that the pattern of whiG segregation determined the frequency of whi recombinants. These data confirmed the genotype of the whiAG strain. Very similar data were obtained in crosses with the whiAH and $A I$ strains, confirming their genotypes.

Assuming negligible multiple crossing-over, all whi recombinants should have the whiA mutation, and some should also have the whiG mutation. The whiG mutation, however, should only be found among $\mathrm{cys}^{+}$recombinants. Since the phenotype of the parental double mutant was identical to that of a whiG mutant, it follows that some $c y s^{+}$recombinants (i.e. those carrying whiA and whiG) should have the whiG (type I) phenotype, and the rest the whiA (type II) phenotype, while all $c y s^{-}$recombinants should have the type II phenotype. This was indeed observed (Table 2), confirming the epistasis of whiG over whiA. Similarly, with the equivalent whiAH double mutant test cross, segregation of aerial mycelium phenotype was observed only among $c y s^{+}$recombinants (3 type II, 6 type III) while five $c y s^{-}$ 


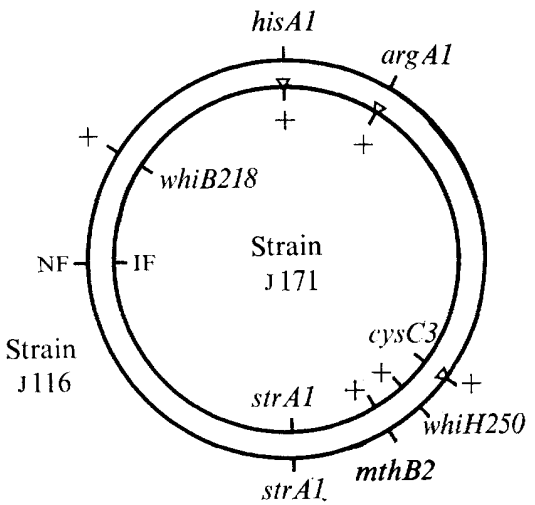

(a)

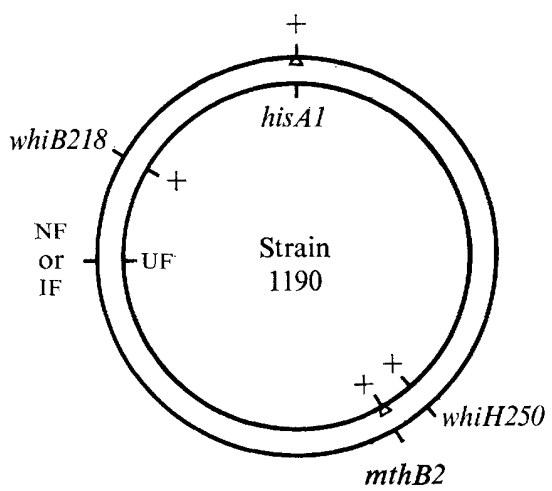

(b)

Fig. 4. Synthesis of a whiBH double mutant. (a) Strains J I 6 and JI 7I were crossed and $h i s A^{+} \arg A^{+}$ cys $C^{+}$recombinants selected (triangles). These were screened for homoserine requirement (the $m t h B 2$ phenotype). (b) Patches of a number of $m t h B 2$ recombinants were then plate-crossed with strain I 190 and the plate crosses replica-plated to medium lacking histidine and homoserine. The frequency of recombinants and their aerial mycelium colour (grey or white) was scored, allowing the identification of IF recombinants (which would be expected to possess the whiB218 mutation) and of those NF recombinants that possessed the whiB218 mutation. For further explanation, see text. $w h i B G$ and $B I$ double mutants were obtained by the same procedure, but substituting strains J90 or JI59 for JII6.

recombinants tested were all type II. This confirmed the epistasis of whiH over whiA. The prediction was different for the equivalent whiAI double mutant test cross: since all whi segregants should carry the whiA mutation and whiA appears epistatic to whil, segregation of aerial mycelium type was not expected. This was the case, all of nine $c y s^{+}$and two $c y s^{-}$ recombinants having the type II phenotype.

Similar consideration of the diagram in Table 3 leads to the prediction that, for the whiAG and $A H$ crosses, all whi recombinants should have the whiG or whiH phenotype. However, in the whiAI cross all cysD his $A$ whi and most cys ${ }^{+}$his $A$ whi recombinants should inherit only the whiI mutation and hence the type IV phenotype, while a few cys ${ }^{+}$his $A$ whi recombinants, and all $\mathrm{cys}^{+} \mathrm{his}^{+}$recombinants, should also have the whiA mutation and hence the epistatic type II phenotype. These predictions were confirmed, no segregants of type II phenotype being observed with the whiAG or $A H$ crosses, while the expected segregation of type II and IV phenotypes occurred in the whiAI cross. In this latter cross all $23 c y s^{+} h i s^{+}$whi recombinants tested were type II, while only one of $16 c y s^{+}$his $A$ whi recombinants tested was type II, the rest being type IV.

It is concluded from these results that the proposed genotypes of the prospective double whi mutants were correct, that whiG and $H$ are epistatic to whiA, and that whiA is epistatic to whil.

\section{Synthesis and testing of whiBG, BH and BI double mutants}

As in the previous section, data for only one double mutant are given in detail (Fig. 5), similar data for the other strains being mainly summarized in the text.

Fig. $4 a$ illustrates the cross used to synthesize a whiBH double mutant. Ignoring multiple crossing-over, strains inheriting whiH250 could have inherited whiB2I 8 as well. To identify those that did, $m t h B 2$ recombinants (arrayed in patches on master plates) were replicaplated to a lawn of spores of UF strain I I90. After a period of growth, these 'plate crosses' 


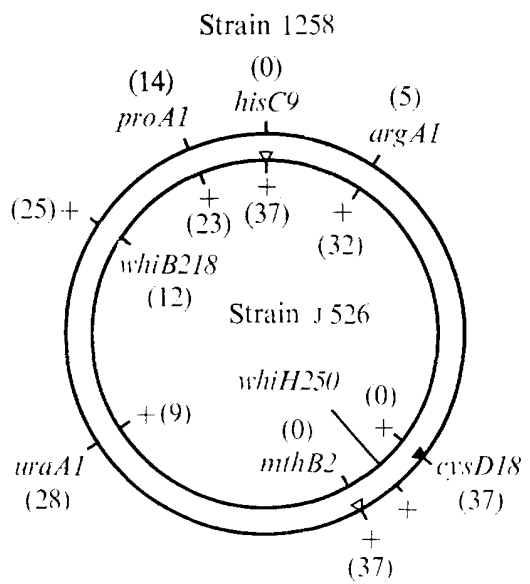

(a)

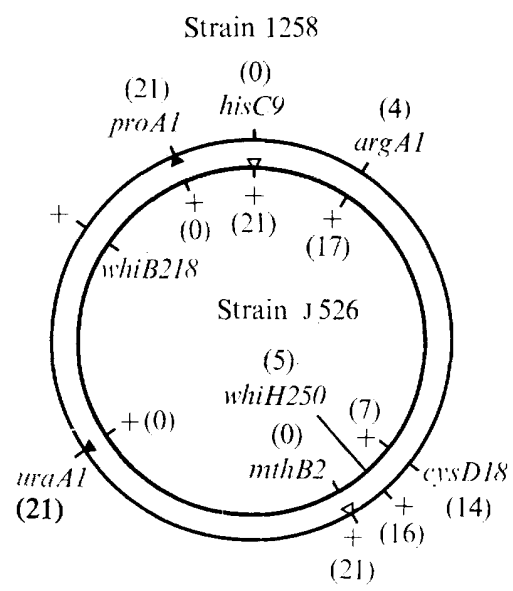

(b)

Fig. 5. Analysis of a strain (J526) carrying whiB and $H$ mutations. Strains $\mathrm{J}_{526}$ and 1258 were crossed and selection (open triangles) made for $h i s A^{+} m t h B^{+}$recombinants. These were analysed for non-selective markers, and samples of whi recombinants re-streaked and examined microscopically. (a) The allele frequencies obtained by considering only $c y s D$ recombinants (additional selection indicated by black triangle) which are all presumed to be $w_{h i H}{ }^{+}$but are free to segregate for whiB2I 8 or whiB ${ }^{+}$. All the cys $D$ whi recombinants tested had the whiB (type II) phenotype, (b) The allele frequencies obtained by considering only pro $A$ ura $A$ recombinants (black triangles) which are all presumed to be $w h i B^{+}$but are free to segregate for whiHzso or whiH ${ }^{+}$. All the pro A uraA whi recombinants tested had the whiH (type III) phenotype.

were replica-plated to selective medium lacking histidine and homoserine (Fig. $4 b$ ). This allowed the identification of any IF strains among the patches on the master plate, as these gave few if any recombinants with the tester strain. It can be seen (Fig. $4 a, b$ ) that such IF recombinants should have inherited the whiB2I 8 mutation. The plate cross with strain I I90 also allowed the identification of NF strains carrying the whiB2I 8 mutation. Selection for $h i s^{+}$in the plate cross ensured that the whiB region of any NF strain tested should be inherited by most of the recombinants, while the selection for $m t h B^{+}$, together with the asymmetrical nature of zygote structure in NF $\times$ UF crosses (Hopwood, Harold, Vivian \& Ferguson, 1969) ensured that the $w_{h i H^{+}}$allele of strain I I90 would be inherited by almost all recombinants. Thus the predominant colour, white or grey, of the aerial mycelium of the recombinant patches after 4 days' incubation indicated the presence or absence of the whiB2I 8 mutation in the appropriate patch on the master plate. The whiBH double mutant chosen for study, J526, was NF (Fig. 5), as was the whiBI double mutant used, J953 (Table 4). The whiBG double mutant studied, however, was IF. (The fertility type of these recombinants had significance only for genetic analysis, and did not affect morphology).

The presence of the expected whi mutations in the double mutants was confirmed by crossing with strain I 258 and, for the whiBI double mutant, also with strain I I07. In the crosses with strain $\mathbf{I} 258$ (Fig. 5), the selection was different from that described earlier (Table 2) for whi $A G, A H$ and $A I$ double mutants, since the two whi mutations were separated by the points of selection and segregated independently to a large extent. To show that whiB2r 8 was present, only recombinants requiring cysteine were considered (Fig. $5 a$ ), since the $\mathrm{whiH}^{+}$allele of strain 1258 would be present in nearly all these progeny. Among these, a whi mutation giving the whiB2I 8 phenotype was found to segregate in a way consistent 
Table 4. Further analysis of a strain (J953) carrying whiB and I mutations

Strains $\mathrm{J} 953$ and 1107 were crossed, and selection (triangles) made for $m t h B^{+}$leu $B^{+}$recombinants. For further details, see Tables 2 and 3 and text.

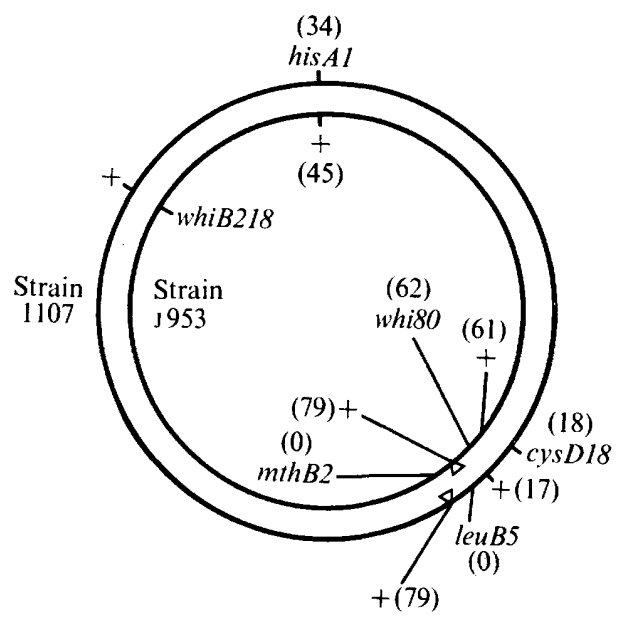

\begin{tabular}{lccc}
\multicolumn{1}{c}{$\begin{array}{c}\text { Frequency } \\
\text { of }\end{array}$} & $\begin{array}{c}\text { Phenotype of aerial mycelium } \\
\text { in a sample of recombinants }\end{array}$ \\
his $A$ cys $D$ & recombinants & Type II & Type IV \\
his $A$ cys $D$ whi & 17 & - & - \\
his $A$ whi & I & - & I5 \\
whi & I6 & I5 & 5 \\
& 45 & &
\end{tabular}

only with a location between proA and uraA. Conversely, considering only pro $A$ ura $A$ recombinants, nearly all of which should be whiB $B^{+}$(Fig. $5 b$ ), a whi mutation having the whiH25o phenotype segregated in a way consistent only with a map location in the $m t h B$ cys $D$ interval. Thus the presence of the two whi mutations was confirmed in a single cross. In addition, the aerial mycelium phenotype of $\operatorname{proA}^{+} u \mathrm{raA} A^{+}$(and hence whiB2I8) recombinants was examined. All those inheriting cys $D$ should be $w h i H^{+}$and therefore have the type II (whiB2I8) phenotype. However, a proportion of the cys $s^{+}$recombinants should inherit the whiH25o mutation and therefore should have the type III phenotype shown by the parental double mutant (strain J526; Table I, Fig. 2). These predictions were confirmed; all three $c y s D$ recombinants scored were type II, as compared with only two out of $32 c y s D^{+}$, the rest of which were type III. This substantiated the earlier conclusion that whiH was epistatic to whiB.

This kind of analysis gave a similar and equally unambiguous result with the whiBG mutant, confirming its genotype and the epistasis of whiG over whiB. With the whiBI double mutant, too few recombinants of the appropriate genotype were obtained in the cross with strain 1258 to confirm the presence of whil8o, though the presence of whiB2I 8 was amply confirmed. A further cross, with strain 1 107, was therefore used (Table 4). The pattern of white colony segregation was compatible only with the presence in strain 3953 of a whi mutation between the leuB and cys $D$ genes, i.e. in the region of the whil gene. The morphology of whi recombinants was also examined, in order to test the predicted pattern of 


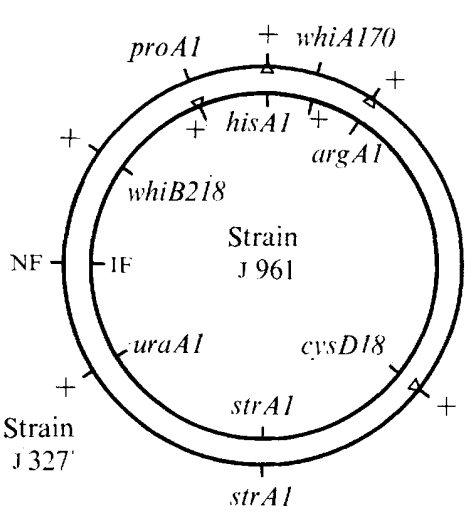

(a)

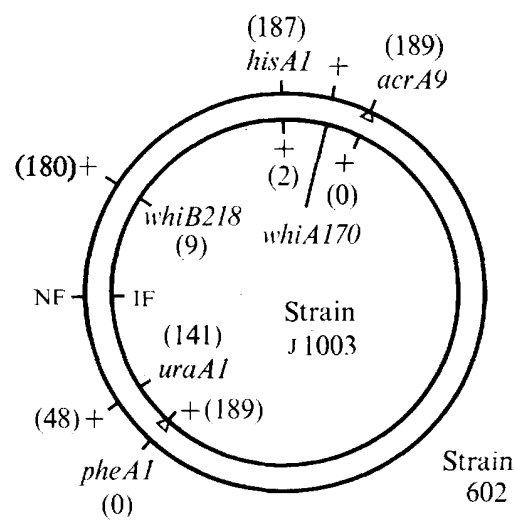

(b)

Fig. 6. The synthesis of a whiAI70 whiB2I8 double mutant. (a) Strains J327 and J96I were crossed and selection (triangles) made for proA $A^{+}$his $A^{+} \arg A^{+} c y s D^{+}$recombinants, most of which should also have inherited whiAI70 from strain $\mathbf{3} 327$. The recombinants were classified for fertility (IF or $\mathrm{NF}$ ) and uracil requirement, and seven $u \mathrm{ra}^{-}$IF recombinants picked. Most of these should have inherited whiB2I 8 from strain J96I. The presence of whiAI7o was confirmed by the absence of whi ${ }^{+}$recombinants in a backcross with strain $\mathrm{J} 327$ (see text). (b) For confirmation of the presence of whiB2I 8 , strain $\mathrm{JI}^{003}$ was crossed with strain 602 and selection (triangles) made for phe $A^{+}$acr $A$ (acriflavine-resistant) recombinants. After recombinant analysis, the nine whi recombinants were re-streaked and examined microscopically. All were of phenotype II, and they included one carrying hisAr. Allele frequencies are given on the diagram.

segregation. The whiBI parent strain (J953) had type II (whiB) morphology (Table I, Fig. 2), so that the presence or absence of whiB2I 8 rather than whil8o should have been decisive in controlling the phenotypes of recombinants. This led to the prediction that a proportion of the $h i s A^{+}$recombinants should have been type II, all other whi recombinants being type IV. This was confirmed (Table 4).

\section{Synthesis and testing of $a$ whiAB double mutant}

The cross used to synthesize a strain carrying whiAI70 and B2I8 is illustrated in Fig. $6 a$. The presence of whiAI7O, in seven presumptive double mutants, was confirmed by backcrossing to the parent whiAI70 strain $\mathrm{J}_{327}$. In the case of strain J1003, finally selected, all Ioo re-streaked recombinants were white. Similarly, no grey recombinants were observed with any of the other six strains. The presence of whiB2I 8 in strain $\mathrm{JIOO}_{3}$ was confirmed by a cross (Fig. $6 b$ ) in which the white/grey colony colour segregated with a frequency indicating the presence of a whi mutation located between the his $A$ and uraA genes.

\section{Epistasis among whiG, $H$ and $I$}

The whiG, $H$ and $I$ loci are all situated between $l e u B$ and cys D (Fig. I) (Chater, 1972), and with the currently available methods of gene transfer in $S$. coelicolor it would be exceedingly arduous to make and confirm whiGH, GI and $H I$ double mutants. A different method was therefore used to test epistasis. The frequency of different mutant phenotypes was scored among the progeny of crosses where the double whi genotype was expected to occur with a significant and predictable frequency. This situation was provided in a series of crosses made between cys $D_{I} 8$ (or in one case $c_{3 s} C_{3}$ ) and leuB5 mutants carrying whi ${ }^{+}$, whiG7I, $H 250$ or $I I 7$, with selection for leu $u^{+} y s^{+}$recombinants (Table 5).

In crosses homozygous for any whi allele, only whi (never $w h i^{+}$) recombinants arose, and 


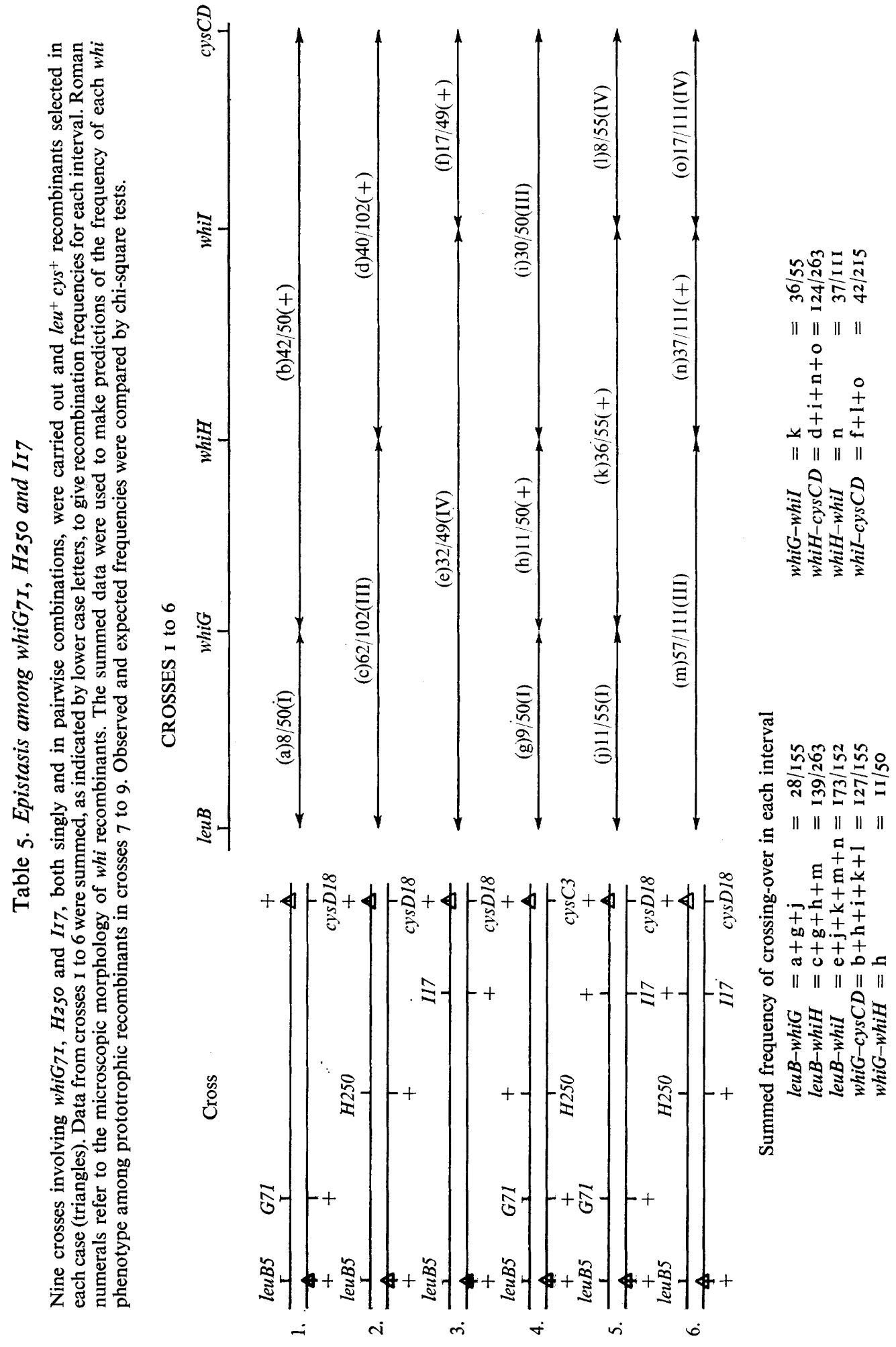




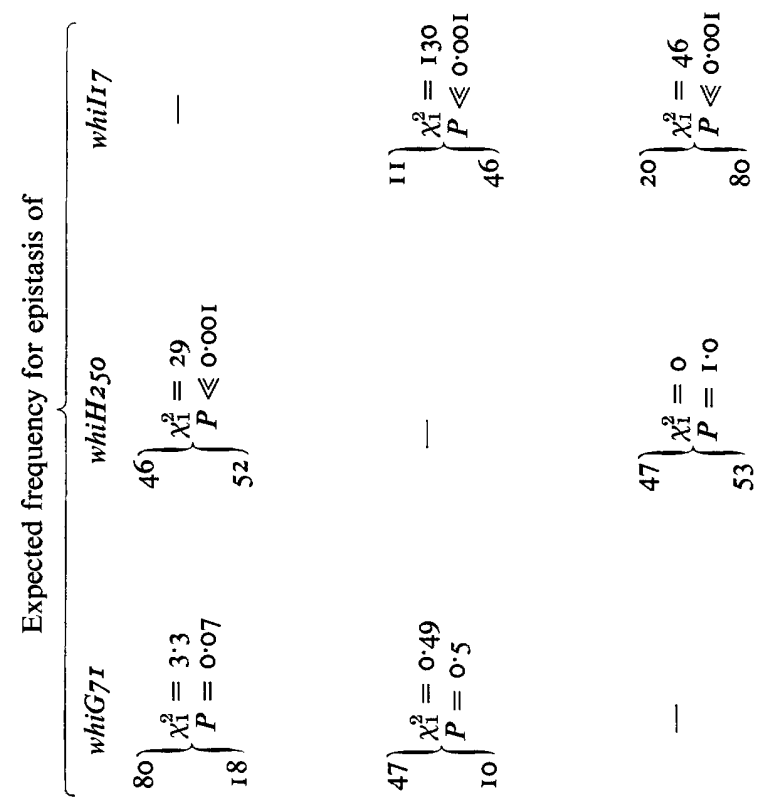

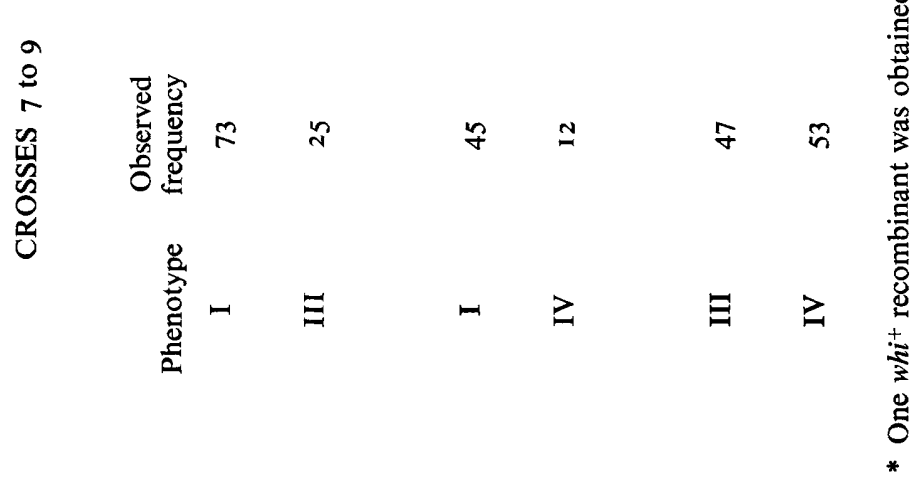

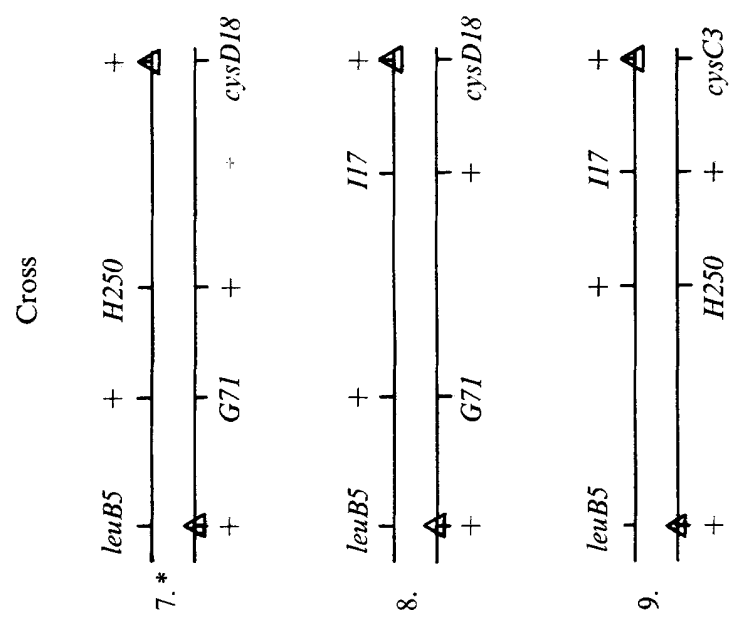


these were all of the expected microscopic phenotype (data not given). This confirmed the validity of the scoring of the phenotypes. In crosses I to 3 of Table 5, only the leuB5 parent was a whi mutant. From these crosses were derived estimates of the relative frequencies of crossing-over in the leuB-whi and whi-cysD intervals. The validity of the scoring of microscopic phenotypes was again upheld.

Crosses 4 to 9 of Table 5 were heterozygous for all three possible pairwise combinations of whi mutations, each in both coupling arrangements with the selective markers. For example, crosses 4 and 7 involved whiG7I and $H_{250}$ in alternative coupling arrangements. Ignoring rare multiple crossover classes, in cross 4 frequent $w h i^{+}$recombinants were generated by crossing-over between whiG and $H$, while crossing-over in the same region in cross 7 generated whiG whiH recombinants. Assuming no unknown selective effects, these two classes should be equally frequent. Three possible phenotypes can be envisaged for a given double whi mutant: like one whi parent, like the other, or different from both. In the present crosses, only the parental phenotypes were observed; thus the double mutants always resembled one of the parent strains. From the relative frequencies of the two whi phenotypes segregating in the cross this parent could be determined, with the result that whiG was found to be epistatic to whiH and $I$, and whiH to whiI. In each case very good agreement of the observed segregation with that expected from epistasis of one of the whi genes was obtained (Table 5).

\section{DISCUSSION}

The main conclusions arising from this work are: (i) the aerial mycelium of double whi mutants for the whiA, B, G, $H$ and $I$ genes is always morphologically similar to one of the single mutant parent strains; (ii) a consistent scheme of epistasis emerges, with whiG epistatic to whiA, $B, H$ and $I$, whiH to whiA, $B$ and $I$, and whiA and $B$ to whil. Thus no complex interactions were detected.

It is difficult to make definite interpretations of these results, although we may reasonably state that, directly or indirectly, the whiG gene product causes limited coiling, and the whiH product further coiling, of aerial hyphal cells, and that the whiA and $B$ gene products are perhaps more directly involved in sporulation septation. It is certainly not reasonable to assume that the epistatic scheme reflects the temporal sequence of either gene expression or gene product activity in any simple way.

Combining all the available information on these whi genes, a very speculative model can be put forward. On this model, the whiG and $H$ gene products would be involved in cell wall changes required for the initiation and localization of sporulation septation. The coiling observed would be a secondary consequence of these changes. The whi $A$ and $B$ gene products might be directly involved as structural or catalytic elements in the development of sporulation septa. Since this scheme takes away from the whil gene product the task of regulating the spacing of sporulation septa, it may be that the whil effect upon spacing (Hopwood et al. 1970) is indirect and might result from, for example, an effect upon the distribution of chromosomal material to spore compartments that occurs during normal sporulation septation.

I am grateful to Judy Elsey-Warren and Lynn Pearse for their skilled and extensive assistance and to David Hopwood and Mike Merrick for helpful comments on the manuscript. 


\section{REFERENCES}

CHATER, K. F. (I972). A morphological and genetic mapping study of white colony mutants of Streptomyces coelicolor A3(2). Journal of General Microbiology 72, 9-28.

Chater, K. F. \& Hopwood, D. A. (I973). Differentiation in Actinomycetes. Symposia of the Society for General Microbiology 23, 143-160.

Chater, K. F. \& Merrick, M. J. (I974). Approaches to the study of differentiation in Streptomyces coelicolor A3(2). In Proceedings of the Second International Symposium, Genetics of Industrial Microorganisms. London: Academic Press.

Hopwood, D. A. (1959). Linkage and the mechanism of recombination in Streptomyces coelicolor. Annals of the New York Academy of Sciences 81, 887-898.

Hopwood, D. A., Chater, K. F., Dowding, J. E. \& Vivian, A. (1973). Advances in Streptomyces coelicolor genetics. Bacteriological Reviews 37, 371-405.

Hopwood, D. A., Harold, R. J., Vivian, A. \& Ferguson, H. M. (I969). A new kind of fertility variant in Streptomyces coelicolor. Genetics $62,461-477$.

Hopwood, D. A., Wildermuth, H. \& Palmer, H. M. (I970). Mutants of Streptomyces coelicolor defective in sporulation. Journal of General Microbiology 6r, 397-408.

MCVITTIE, A. (1974). Ultrastructural studies on sporulation in wild-type and white colony mutants of Streptomyces coelicolor. Journal of General Microbiology 81, 29 I-302.

Vivian, A. \& Hopwood, D. A. (1970). Genetic control of fertility in Streptomyces coelicolor A3(2): the IF fertility type. Journal of General Microbiology 64, IOI-I I 7 .

Wildermuth, H. \& Hopwood, D. A. (1970). Septation during sporulation in Streptomvces coelicolor Journal of General Microbiology 6o, 5I-59. 